\title{
q-Independent Slow Dynamics in Atomic and Molecular Systems
}

\author{
Philip H. Handle, ${ }^{1}$ Lorenzo Rovigatti, ${ }^{1,2,}$ and Francesco Sciortino ${ }^{1}$ \\ ${ }^{1}$ Department of Physics, Sapienza University of Rome, Piazzale Aldo Moro 5, I-00185 Roma, Italy \\ ${ }^{2}$ CNR-ISC, UoS Sapienza, Piazzale Aldo Moro 5, I-00185 Roma, Italy
}

(Received 1 August 2018; published 3 May 2019)

\begin{abstract}
Investigating million-atom systems for very long simulation times, we demonstrate that the collective density-density correlation time $\left(\tau_{\alpha}\right)$ in simulated supercooled water and silica becomes wave-vector independent $\left(q^{0}\right)$ when the probing wavelength is several times larger than the interparticle distance. The $q$ independence of the collective density-density correlation functions, a feature clearly observed in lightscattering studies of some soft-matter systems, is thus a genuine feature of many (but not all) slowdynamics systems, either atomic, molecular, or colloidal. Indeed, we show that when the dynamics of the density fluctuations includes particle-type diffusion, as in the case of the Lennard-Jones binary-mixture model, the $q^{0}$ regime does not set in and the relaxation time continues to scale as $\tau_{\alpha} \sim q^{-2}$ even at small $q$.
\end{abstract}

DOI: 10.1103/PhysRevLett.122.175501

The wave-vector $(q)$ dependence of the dynamics of atomic, molecular, and colloidal systems close to dynamic arrest has been the focus of intense research [1-11]. In particular, the $q$ region that corresponds to the nearest-neighbor distance in glass- and gel-forming systems has revealed a series of interesting phenomena [12,13]: (i) a two-step relaxation for both self- and collective density correlation functions, indicating a faster intracage motion and a slower structural relaxation ( $\alpha$ relaxation), respectively; (ii) a significant stretching of the $\alpha$ relaxation, which originates from the coupling between distinct modes [14,15]; (iii) a deviation from the diffusive $q^{-2}$ behavior of the self-correlation time; (iv) oscillations in the $q$ dependence of the collective relaxation time, often in phase with the oscillation of the structure factor; (v) a faster decay of the self-dynamics compared to the collective one, indicating that relative particle displacements play an important role in the decorrelation of the system.

The region at very small $q$, where the wavelength is much larger than the interparticle distance, has also been thoroughly characterized. Here, conservation laws in onecomponent systems determine a three-mode decay of the collective correlation functions [16]: two modes associated with damped sound waves (the Brillouin peaks) and one to the damped decay of the heat diffusion (the Rayleigh peak). In all three cases, the damping time follows a $q^{-2}$ dependence. In the case of glasses (where the $\alpha$-relaxation time is longer than the experimental observation), a clear crossover has been identified between the region where the system can be considered an elastic continuum and the region where an excess of vibrational states $[17,18]$ is superimposed to the Debye density of states [19-21].

In some colloidal systems, where the size of the particles provides access to smaller ratios between the wavelength of the probe radiation and the interparticle distance, a $q$-independent $\left(q^{0}\right)$ relaxation mode has been reported. From the early measurements in polymer melts in which entanglement induces an effective transient network [22-24], evidence of a $q^{0}$ mode has been presented for rodlike micelles [25], semidilute polymers with bonding agents [26,27], telechelic ionomers [28], and microemulsion droplets in solution with telechelic polymers [29] (where the latter provide transient links between distinct droplets). More recently, a $q^{0}$ mode has been reported for equilibrium gels of DNA tetrafunctional nanostars [30,31] in which a short self-complementary DNA sequence provides a temperature-controllable link between different particles. This $q^{0}$-relaxation dynamics has been interpreted as originating from local elasticity fluctuations propagating through the system [22,31]. A recent simulation study of a particle model for vitrimers, binary-mixture networks in which the microscopic dynamics is slaved to a bondswapping process [32], has also reported a clear $q^{0}$ dependence for the collective dynamics extending over more than one order of magnitude in $q$.

In this Letter, we explore the possibility that a $q^{0}$ dependence of the collective relaxation time is much more common than previously thought, being a generic feature of systems with slow dynamics, including systems composed of atoms and molecules. The $q^{0}$ mode can arise in a range of $q$ values intermediate between the hydrodynamic and the nearest-neighbor regions when the collective relaxation is not associated with single-particle diffusion. We simulate slow-dynamics systems that are large enough (up to a million atoms) to allow the numerical evaluation of the collective relaxation over wavelengths corresponding to distances up to 50 times larger than the typical nearestneighbor distance. Here we mostly focus on supercooled water, whose dynamics has been extensively investigated experimentally with different scattering techniques 
[33-37]. For water, a comparison between the ultrasound data [38] and the lowest $q$ explored with inelastic x-ray scattering [33] suggests that a region with $q^{0}$ dispersion exists just below the experimentally accessible window. Using simulations, we now provide clear evidence that, indeed, there exists a wide range of wave vectors in which the collective dynamics is $q$ independent. We also show that a model based on the Mori-Zwanzig formalism properly describes the $q$ independence of the dynamics. Finally, we present results for two more systems, both well-known binary models of glass formers: the Beest-Kramer-van Santen (BKS) model for silica [39] and a binary-mixture Lennard-Jones (BMLJ) [40]. In agreement with our explanation, we observe a $q^{0}$ mode only in BKS silica.

Methods.-For TIP4P/2005 [41] and BKS [39], we perform NVT simulations utilizing GROMACS 5.1.4 [42] with a velocity-Verlet integrator and a time step of $\Delta t=1 \mathrm{fs}$. The temperature is controlled using a NoséHoover thermostat $[43,44]$. The Coulomb interactions are included with a particle-mesh Ewald treatment [45] with a Fourier spacing of $0.1 \mathrm{~nm}$. For both the Lennard-Jones and real space Coulomb interactions, the same cutoff $r_{\text {cut }}$ is used $\left(r_{\text {cut }}=0.9\right.$ and $1 \mathrm{~nm}$ for TIP4P/2005 and BKS, respectively). Lennard-Jones interactions beyond $r_{\text {cut }}$ are taken into account assuming a uniform fluid density. The TIP4P/2005 system consists of $2.5 \times 10^{5}$ molecules $(1 \mathrm{M}$ interacting sites) in a cubic box. We investigate three densities $\left(0.90,0.95\right.$, and $1.00 \mathrm{~g} / \mathrm{cm}^{3}$ corresponding to box lengths of $20.26,19.89$, and $19.56 \mathrm{~nm}$, respectively) at two temperatures (240 and $250 \mathrm{~K})$. The molecular geometry is maintained using the LINCS (linear constraint solver) algorithm [46]. The BKS system consists of 287 712 particles $(1 / 3 \mathrm{Si}$ and $2 / 3 \mathrm{O})$ in a cubic box. We study three densities $\left(2.36,2.48\right.$, and $2.60 \mathrm{~g} / \mathrm{cm}^{3}$, corresponding to box lengths of $15.95,15.69$, and $15.45 \mathrm{~nm}$ ) at $3500 \mathrm{~K}$. As a BMLJ glass former, among the different alternatives [47-49], we choose the Kob-Andersen model [40]. We use the RUMD package [50] to simulate $10^{6}$ particles at $\rho^{*}=1.2$ and $T^{*}=0.466$, where the ${ }^{*}$ superscript indicates reduced units: The unit of length is the diameter of the large particles, $\sigma_{A A}$, the unit of mass is the particle mass $m$, and the unit of energy is the interaction strength between large particles, $\epsilon_{A A}$. Differently from Ref. [40], the unit of time is $\sqrt{m \sigma_{A A} / \epsilon_{A A}}$. The total duration of the simulations is $20 \mathrm{~ns}$ for TIP4P/2005 and BKS (20-100 times the slowest collective relaxation time in the system) and $6 \times 10^{7}$ MD time steps for the BMLJ system. The relaxation times and nonergodicity factors obtained from fitting the correlation functions of the TIP4P/2005 and BKS systems in the whole $q$ range are reported in Supplemental Material [51].

Results: TIP4P/2005.-Figure 1 shows the (oxygenoxygen) collective density correlation functions $F(q, t)$ for several $q$ values in the window $0.3<q<3 \mathrm{~nm}^{-1}$, corresponding approximatively to wavelengths $7-70$ times
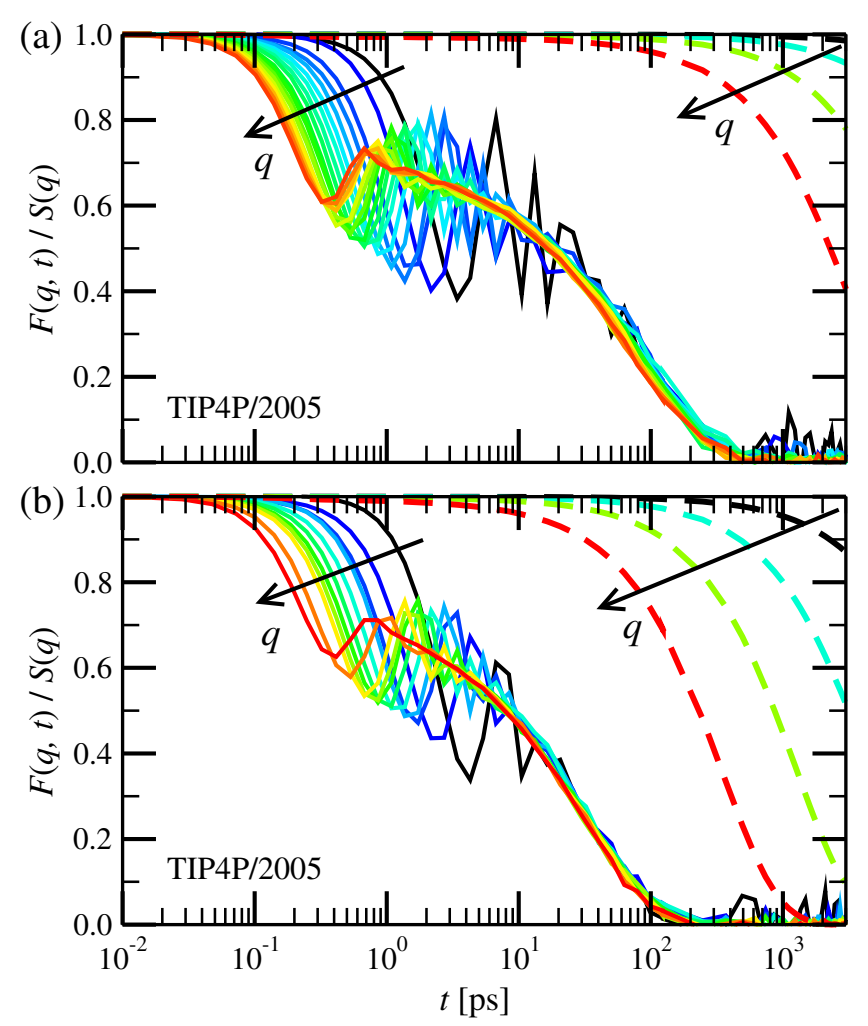

FIG. 1. Decay of the (full lines) collective and (dashed lines) self-density fluctuations at (a) $T=240 \mathrm{~K}, \rho=0.9 \mathrm{~g} / \mathrm{cm}^{3}$ and (b) $T=250 \mathrm{~K}, \rho=1.0 \mathrm{~g} / \mathrm{cm}^{3}$. In both panels, $q$ varies between 0.3 (black lines) and 3.0 (red lines) $\mathrm{nm}^{-1}$.

larger than the $\mathrm{OO}$ nearest-neighbor distance $(0.28 \mathrm{~nm})$. $F(q, t)$ is evaluated as

$$
F(q, t)=\left\langle\rho_{\mathbf{q}}^{*}(t) \rho_{\mathbf{q}}(0)\right\rangle, \quad \rho_{\mathbf{q}}(t) \equiv \frac{1}{\sqrt{N}} \sum_{i=1}^{N} e^{i \mathbf{q} \cdot \mathbf{r}_{i}(t)},
$$

where $N$ is the total number of molecules, $\mathbf{r}_{i}$ is the position of the $i$ th oxygen atom, and the average is performed over different initial times along the MD trajectory and over all $\mathbf{q}$ vectors with the same modulus $q . F(q, 0)$ coincides with the structure factor $S(q)$. It is immediately clear from Fig. 1 that, in this $q$ window (extending the window of $q$ explored with high-resolution inelastic x-ray scattering measurements $[33,34])$, all correlation functions decay at long times with the same characteristic timescale. Figure 1 also shows the self-correlation function $F_{\text {self }}(q, t)$, plotted with dashed lines. In this small- $q$ region, the Gaussian approximation holds true; that is, $F_{\text {self }}(q, t)=\exp \left(-q^{2}\left\langle r^{2}(t)\right\rangle / 6\right)$, where $\left\langle r^{2}(t)\right\rangle$ is the mean-squared displacement [16] (comparison not shown). It is important to note that the self-correlation functions decay at much longer times compared to $F(q, t)$, indicating that the decorrelation of the collective dynamics over the probed length scales does not require particle diffusion. 
To further support the idea that a single ( $q$-independent) characteristic time controls the decay of the density fluctuations in this low- $q$ region, we compare the numerically calculated correlation functions with predictions based on the Mori-Zwanzig (MZ) formalism. For any system, the normalized collective correlation function $\phi_{q} \equiv F(q, t) / S(q)$ formally satisfies the equation [16]

$$
\frac{d^{2} \phi_{q}}{d t^{2}}+\Omega_{q}^{2} \phi_{q}+\int_{0}^{t} M_{q}\left(t-t^{\prime}\right) \frac{d \phi_{q}}{d t} d t^{\prime}=0
$$

where $\Omega_{q}^{2}=\left(k_{B} T / M\right)\left[q^{2} / S(q)\right]$.

The memory function, which is the autocorrelation of the stochastic force [16], and $\Omega_{q}^{2}$ completely specify the dynamics. Guided by the $q$ independence of $\tau_{\alpha}$, we approximate $M_{q}(t)$ as

$$
\begin{aligned}
& M_{q}(t)=\gamma_{0} q^{2} \delta(t)+M_{q}^{s}(t), \\
& M_{q}^{s}(t) \equiv A_{s} q^{2} \exp \left[-\left(t / \tau_{s}\right)^{\beta}\right],
\end{aligned}
$$

where $\gamma_{0} q^{2} \delta(t)$ describes the damping associated with the fast microscopic dynamics and $M_{q}^{s}(t)$ models the structural relaxation. This function is characterized by the three parameters $A_{s}, \tau_{s}$, and $\beta$, defining, respectively, the amplitude and the time dependence of $M_{q}^{s}(t)$. All four parameters $\left(\gamma_{0}, A_{s}, \tau_{s}\right.$, and $\left.\beta\right)$ are, in principle, functions of $q$. Consequently,

$$
\frac{d^{2} \phi_{q}}{d t^{2}}+\Omega_{q}^{2} \phi_{q}+\gamma_{0} q^{2} \frac{d \phi_{q}}{d t}+\int_{0}^{t} M_{q}^{s}\left(t-t^{\prime}\right) \frac{d \phi_{q}}{d t} d t^{\prime}=0 .
$$

In deriving Eq. (4), we have neglected the coupling between density and temperature fluctuations and the thermal diffusion contribution which is (in the hydrodynamic limit) proportional to $C_{v} / C_{p}-1$ [54]. For water, this is a reasonable approximation, since at the temperature of maximum density $C_{v}=C_{p}$ [54].

We solve the time dependence of Eq. (4) numerically (in Fourier space), searching for the values of the parameters which minimize the differences with the correlation functions evaluated from the simulated trajectories. We find that, in the range $0.3<q<3 \mathrm{~nm}^{-1}$, it is possible to represent all correlation functions in the entire time window by using $q$-independent values for $\gamma_{0}, A_{s}, \tau_{s}$, and $\beta$. Figure 2 compares the $q$-independent model predictions with the numerical data for two different state points. Such excellent agreement provides strong evidence that a collection of local relaxation processes (as indicated by the value $\beta=0.5$ ) fully describes the $q$-independent decay of the collective dynamics at small $q$. The prediction of Eq. (4) for $q$ smaller than the ones numerically accessed [black lines in Fig. 2(b)] visually demonstrates the crossover to the hydrodynamic limit (having neglected the $T$ fluctuations,
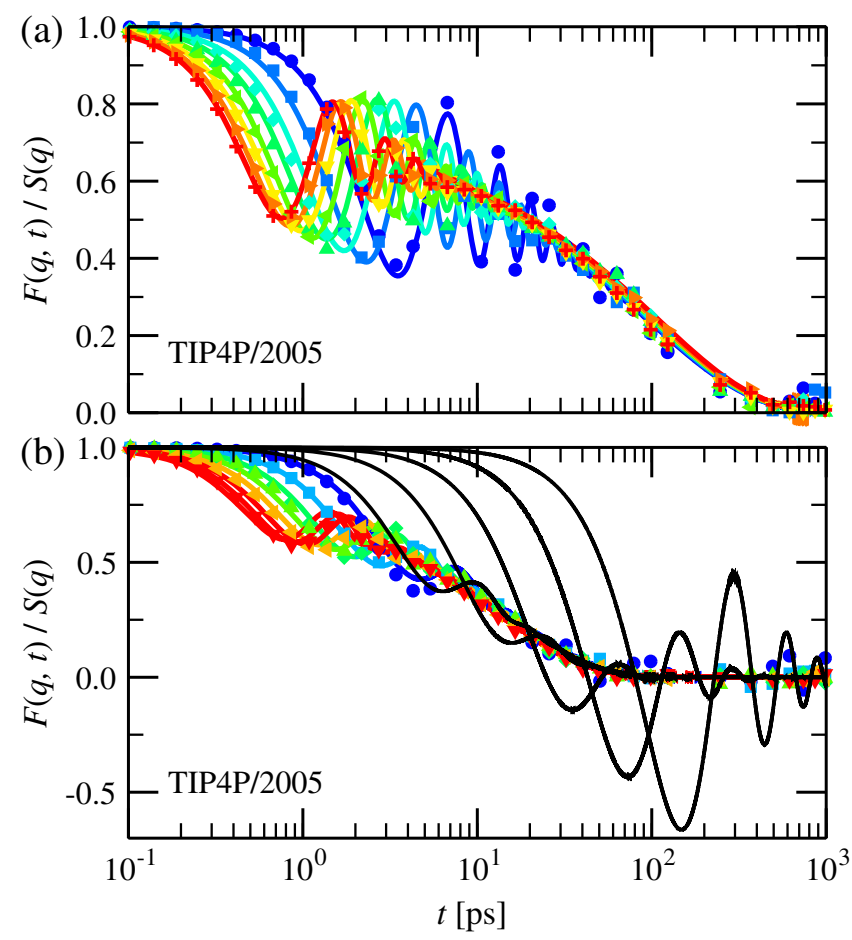

FIG. 2. Simulation (symbols) and theoretical (lines) results for (a) $\rho=0.90 \mathrm{~g} / \mathrm{cm}^{3}$ and $T=240 \mathrm{~K}$ and (b) for $\rho=1.0 \mathrm{~g} / \mathrm{cm}^{3}$ and $T=250 \mathrm{~K}$, in the same $q$ window as in Fig. 1. For the prediction of Eq. (4), in (a) $\gamma_{0}=0.4$ and $M_{q}^{s}(t)=7.3 q^{2} \exp$ $\left[-(t / 15)^{0.5}\right]$, while in (b) $\gamma_{0}=0.3$ and $M_{q}^{s}(t)=10 q^{2} \exp$ $\left[-(t / 1.05)^{0.5}\right]$ (with $t$ in picoseconds, $q$ in $\mathrm{nm}^{-1}, \gamma_{0}$ in $\mathrm{ps}^{-1} \mathrm{~nm}^{2}$, and $M_{q}$ in $\mathrm{ps}^{-2}$ ). In (b), the black lines show predictions for very small $q$ vectors (from right to left, $q_{n}=$ $0.0016 \times 2^{n-1} \mathrm{~nm}^{-1}$ with $\left.n=1,2,3,4,5\right)$, to highlight the crossover from structural relaxation to hydrodynamics.

the limit coincides with the damped harmonic oscillator model with adiabatic sound speed $\left.v_{s}=\Omega_{q} / q[15,16]\right)$. In general, hydrodynamics sets in when the timescale of the nonstructural component becomes comparable with $\tau_{\alpha}$. Selecting the period of the sound wave as the typical time, the wave vector below which the relaxation time loses its $q^{0}$ character is reached when $q \ll 2 \pi /\left(v_{s} \tau_{\alpha}\right)$. Therefore, upon approaching the glass transition, as $\tau_{\alpha}$ becomes longer and longer, the region of $q$ in which a $q^{0}$ mode is expected becomes larger and larger, provided that $S(q)$ does not vary significantly.

Results: BKS and BMLJ.-We next investigate two of the most commonly studied binary-mixture glass-former models. BKS silica [39] generates a structure in which the (positively charged) $\mathrm{Si}$ atoms form a prevalently tetrafunctional open network in which the (negatively charged) $\mathrm{O}$ atoms mediate the $\mathrm{Si}-\mathrm{Si}$ bonds. As expected for charged systems, there are essentially no large wavelength concentration fluctuations [55]. By contrast, BMLJ [40] generates a dense structure in which each particle is surrounded by particles of both species. BMLJ particles are electrically 


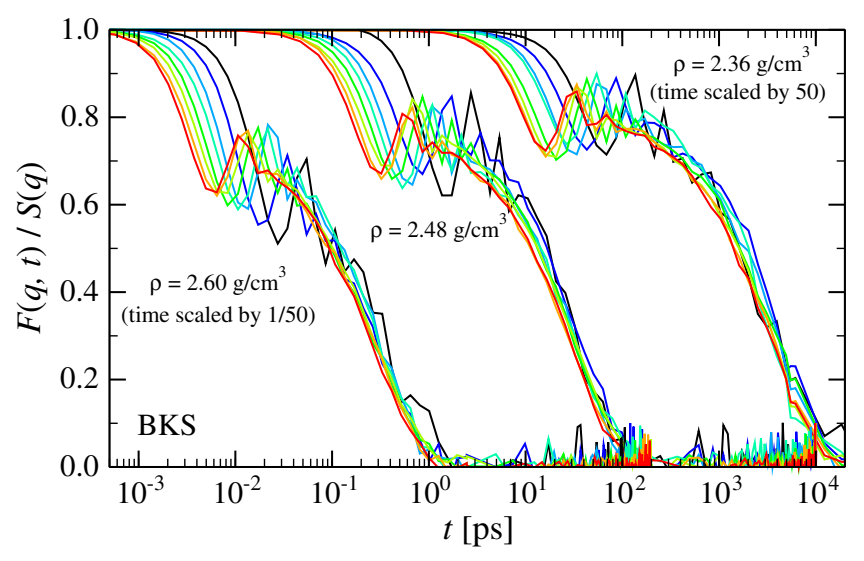

FIG. 3. Decay of the collective number density fluctuations in BKS silica at $T=3500 \mathrm{~K}$ for different densities. In all plots, $0.4<q<2 \mathrm{~nm}^{-1}$ (from black to red). Note that, for the sake of clarity, the time associated with the $\rho=2.60 \mathrm{~g} / \mathrm{cm}^{3}$ and $\rho=$ $2.63 \mathrm{~g} / \mathrm{cm}^{3}$ data have been multiplied by $1 / 50$ and 50 , respectively. As a reference, the $\mathrm{Si}-\mathrm{Si}$ distance is $\approx 0.31 \mathrm{~nm}$.

neutral, and concentration fluctuations do not vanish in the small- $q$ limit. This crucial difference is evident in the Bhatia-Thornton concentration-concentration structure factors [56] (reported in Supplemental Material [51]). In the small- $q$ region explored in the simulation, the decay of the collective total-density fluctuations in the two binary systems is completely different. While BKS (Fig. 3) behaves as TIP4P/2005 water and displays an almost $q^{0}$ dependence for all three investigated densities, the same function in BMLJ manifests a strong $q^{-2}$ dependence [see the main panel and inset in Fig. 4(a)]. We also show the (non-normalized) partial correlation functions for $q \sigma_{A A}=$ 0.4 in Fig. 4(b). All the correlation functions decay similarly in the whole $q$ range considered, and the associated relaxation times always display a $q^{-2}$ dependence. Thus, in contrast to the one-component water case and the two-component silica case, the small- $q \alpha$-relaxation process in BMLJ does not acquire a $q$-independent behavior.

Discussion and conclusions.-The reported investigation of the low- $q$ collective dynamics in atomic and molecular systems exhibiting slow dynamics provides indisputable numerical evidence of the existence of a large region of $q$, corresponding to wavelengths larger than the interparticle distance, in which the relaxation time is $q$ independent. These findings demonstrate that a $q^{0}$ mode is not a prerogative of soft-matter systems, but it is a genuine feature of many slow-dynamics systems, either atomic, molecular, or colloidal. The $q$ independence of $F(q, t)$ can be equivalently expressed as a $q$ independence of the memory function within the MZ formalism.

Interestingly, from the point of view of the mode coupling theory (MCT), which neglects crystallization, the difference between the $q$ dependence of the memory functions associated with the structural relaxation of disordered one-component systems and binary mixtures can

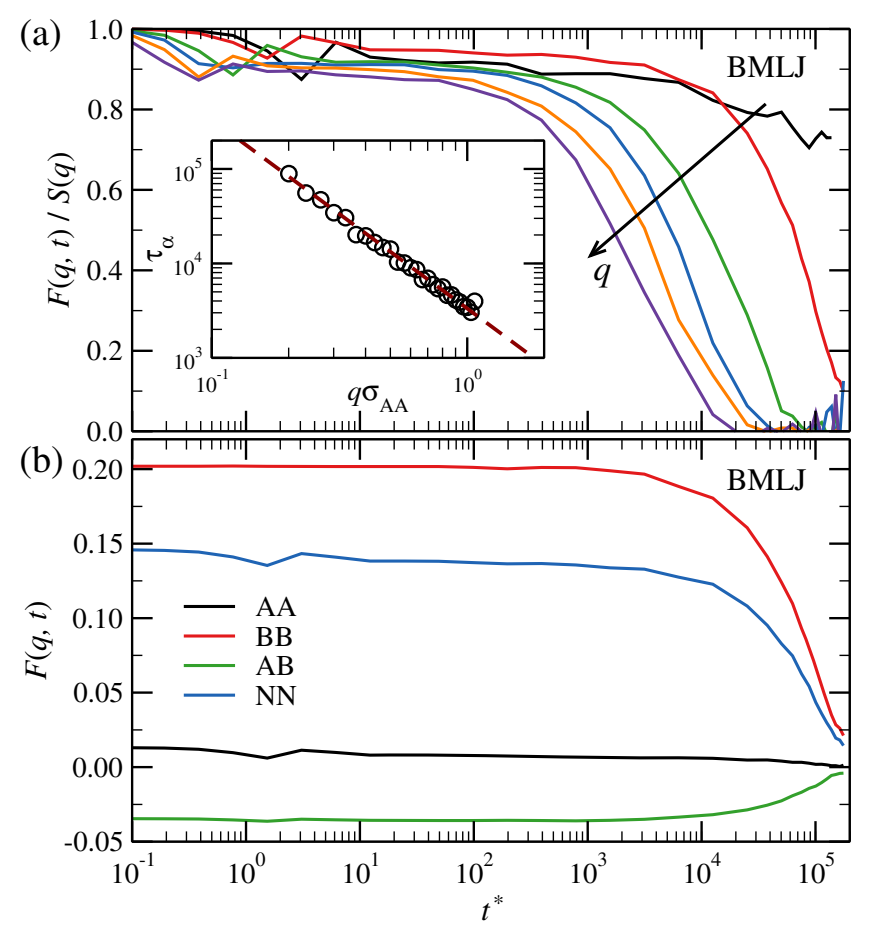

FIG. 4. Density-density collective correlation functions for BMLJ at $T^{*}=0.466$ and $\rho^{*}=1.2$. (a) $F(q, t)$ calculated for all particles, normalized by the structure factor $S(q)$. From right to left, $q_{n} \sigma_{A A}=0.06676 \times n$ with $n=3,6,12,18,24,30$. The inset shows the corresponding relaxation time $\tau_{\alpha}$ as a function of $q$. The dashed line, which has a slope given by $q^{-2}$, is shown for comparison. (b) The non-normalized collective correlation functions calculated for $q \sigma_{A A}=0.4$ between $A A, A B, B B$ and all particles $(N N)$.

be traced back to the conservation of the overall momentum of the system [14,57]. In the one-component MCT, consistent with what we have found, $M_{q}$ is predicted to be $q$ independent at small $q$. In the case of binary mixtures, momentum conservation is not observed by each component, since only the sum of all partial momenta is conserved. As a result, the MCT relaxation times might display a $q^{-2}$ dependence $[14,57]$. Therefore, within this framework, our results seem to indicate that binary mixtures for which the large-wavelength concentration fluctuations are suppressed behave, at large length scales, as effective one-component systems. A comparison between the kinetic and thermodynamic contributions to the interdiffusion constant of the BMLJ and BKS systems clearly demonstrates the presence of strong cross-correlations only in the latter (see Supplemental Material [51]) [16,58,59]. These predictions have never been carefully tested due to the numerical difficulties of simulating very large systems over very long periods of time. Our results will definitively stimulate novel theoretical analysis to properly frame the $q^{0}$ phenomenon. In particular, it will be interesting to understand in more depth under which conditions binary mixtures do or do not exhibit a $q^{0}$ mode. The reported results 
will also stimulate new small-angle neutron and $\mathrm{x}$-ray experiments, where a confirmation of the predicted behavior can possibly be achieved. The results presently available for water [33,34] (reaching a lowest $q$ of $1 \mathrm{~nm}^{-1}$ ) are consistent with the numerical findings discussed here.

Although the memory function formalism provides a deep formal understanding of the $q$ independence, it does not offer a physical picture of the local processes responsible for probing all different available microstates at small length scales. Different systems will relax locally in different ways, possibly via rototranslational motion (as proposed for water [60]), bond swapping, or bond flickering in networks. Despite these differences, the effect of such local decorrelations on the small- $q$ dynamics is system independent. The local changes of elasticity brought in by these local events possibly propagate via the vibrational modes of the system [31] resulting in the decay of the collective density $(\mathrm{N} / \mathrm{V})$ fluctuations at large distances controlled by changes not in the number of particles $N$ but in the volume $V$.

Finally, we observe that in the $q^{0}$ regime equilibration in systems of very large size can be achieved only if simulations can be run for times longer than $\tau_{\alpha}$ (since $\tau_{\alpha}$ does not grow with decreasing $q$ ). This is not the case for BMLJ (and possibly other binary Lennard-Jones glass formers [47-49]), for which doubling the system size requires 4 times longer equilibration times, making it essentially impossible to generate very large structurally and compositionally equilibrated configurations. Indeed, in these cases the diffusion of individual particles is required to relax frozen-in long wavelength concentration fluctuations. The observed $q^{0}$ mode in binary systems, shown here for BKS and previously for binary-mixture vitrimers [32], can possibly originate from the suppression of the small wave-vector (large wavelength) concentration fluctuations. For BKS, this effect stems from the electrostatic nature of the $\mathrm{Si}$ and $\mathrm{O}$ interactions [55]. By contrast, in the vitrimer system [32], it is the precise stoichiometry of the mixture and the bonding mechanism, which can act only between unlike particles, that prevents the occurrence of small- $q$ concentration fluctuations.

We thank G. Monaco and J. F. Douglas for discussions and J. Russo and C. Scherfler for comments on the manuscript. P. H. H. acknowledges financial support from the Austrian Science Fund (FWF Erwin Schrödinger Fellowship No. J3811 N34). L. R. acknowledges support from the European Research Council (ERC Consolidator Grant No. 681597, MIMIC).

*Corresponding author lorenzo.rovigatti@uniroma1.it

[1] F. Mezei, W. Knaak, and B. Farago, Phys. Scr. T19B, 363 (1987).

[2] W. Van Megen and P. N. Pusey, Phys. Rev. A 43, 5429 (1991).
[3] W. Kob and H. C. Andersen, Phys. Rev. E 52, 4134 (1995).

[4] J. Horbach and W. Kob, Phys. Rev. E 64, 041503 (2001).

[5] F. Sciortino, L. Fabbian, S.-H. Chen, and P. Tartaglia, Phys. Rev. E 56, 5397 (1997).

[6] A. Tölle, Rep. Prog. Phys. 64, 1473 (2001).

[7] R. Torre, A. Taschin, and M. Sampoli, Phys. Rev. E 64, 061504 (2001).

[8] T. Eckert and E. Bartsch, Phys. Rev. Lett. 89, 125701 (2002).

[9] R. Bandyopadhyay, D. Liang, H. Yardimci, D. A. Sessoms, M. A. Borthwick, S. G. J. Mochrie, J. L. Harden, and R. L. Leheny, Phys. Rev. Lett. 93, 228302 (2004).

[10] S. M. Bhattacharyya, B. Bagchi, and P. G. Wolynes, Proc. Natl. Acad. Sci. U.S.A. 105, 16077 (2008).

[11] S. Roldán-Vargas, L. Rovigatti, and F. Sciortino, Soft Matter 13, 514 (2017).

[12] K. Binder and W. Kob, Glassy Materials and Disordered Solids: An Introduction to Their Statistical Mechanics (World Scientific, Singapore, 2011).

[13] E. Donth, The Glass Transition: Relaxation Dynamics in Liquids and Disordered Materials (Springer Science \& Business Media, New York, 2013), Vol. 48.

[14] W. Götze, Complex Dynamics of Glass-Forming Liquids: A Mode-Coupling Theory (Oxford University, New York, 2008), Vol. 143.

[15] U. Balucani and M. Zoppi, Dynamics of the Liquid State (Clarendon, Oxford, 1995), Vol. 10.

[16] J.-P. Hansen and I. R. McDonald, Theory of Simple Liquids: With Applications to Soft Matter (Academic, New York, 2013).

[17] W. Schirmacher, G. Diezemann, and C. Ganter, Phys. Rev. Lett. 81, 136 (1998).

[18] G. Monaco and V. M. Giordano, Proc. Natl. Acad. Sci. U.S.A. 106, 3659 (2009).

[19] A. Tanguy, J. P. Wittmer, F. Leonforte, and J.-L. Barrat, Phys. Rev. B 66, 174205 (2002).

[20] J. Wittmer, A. Tanguy, J.-L. Barrat, and L. Lewis, Europhys. Lett. 57, 423 (2002).

[21] A. Zaccone and E. Scossa-Romano, Phys. Rev. B 83, 184205 (2011).

[22] F. Brochard, J. Phys. France 44, 39 (1983).

[23] M. Adam and M. Delsanti, Macromolecules 18, 1760 (1985).

[24] J. Li, T. Ngai, and C. Wu, Polym. J. 42, 609 (2010).

[25] N. Nemoto, M. Kuwahara, M.-L. Yao, and K. Osaki, Langmuir 11, 30 (1995).

[26] N. Nemoto, A. Koike, and K. Osaki, Macromolecules 29, 1445 (1996).

[27] E. Michel, M. Filali, R. Aznar, G. Porte, and J. Appell, Langmuir 16, 8702 (2000).

[28] R. Johannsson, C. Chassenieux, D. Durand, T. Nicolai, P. Vanhoorne, and R. Jerome, Macromolecules 28, 8504 (1995)

[29] E. Michel, M. Filali, R. Aznar, G. Porte, and J. Appell, Langmuir 16, 8702 (2000).

[30] S. Biffi, R. Cerbino, G. Nava, F. Bomboi, F. Sciortino, and T. Bellini, Soft Matter 11, 3132 (2015).

[31] G. Nava, M. Rossi, S. Biffi, F. Sciortino, and T. Bellini, Phys. Rev. Lett. 119, 078002 (2017).

[32] L. Rovigatti, G. Nava, T. Bellini, and F. Sciortino, Macromolecules 51, 1232 (2018). 
[33] G. Monaco, A. Cunsolo, G. Ruocco, and F. Sette, Phys. Rev. E 60, 5505 (1999).

[34] F. Bencivenga, A. Cunsolo, M. Krisch, G. Monaco, G. Ruocco, and F. Sette, Phys. Rev. E 75, 051202 (2007).

[35] R. Torre, P. Bartolini, and R. Righini, Nature (London) 428, 296 (2004).

[36] A. Taschin, P. Bartolini, R. Eramo, and R. Torre, Phys. Rev. E 74, 031502 (2006).

[37] A. Arbe, P. Malo de Molina, F. Alvarez, B. Frick, and J. Colmenero, Phys. Rev. Lett. 117, 185501 (2016).

[38] C. M. Davis and J. Jarzynski, in The Physics and Physical Chemistry of Water (Springer, New York, 1972), pp. 443-461.

[39] B. W. H. Van Beest, G. J. Kramer, and R. A. Van Santen, Phys. Rev. Lett. 64, 1955 (1990).

[40] W. Kob and H. C. Andersen, Phys. Rev. E 51, 4626 (1995).

[41] J. L. Abascal and C. Vega, J. Chem. Phys. 123, 234505 (2005).

[42] D. Van Der Spoel, E. Lindahl, B. Hess, G. Groenhof, A. E. Mark, and H. J. Berendsen, J. Comput. Chem. 26, 1701 (2005).

[43] S. Nosé, Mol. Phys. 52, 255 (1984).

[44] W. G. Hoover, Phys. Rev. A 31, 1695 (1985).

[45] U. Essmann, L. Perera, M. L. Berkowitz, T. Darden, H. Lee, and L. G. Pedersen, J. Chem. Phys. 103, 8577 (1995).

[46] B. Hess, J. Chem. Theory Comput. 4, 116 (2008).
[47] G. Pastore, B. Bernu, J. P. Hansen, and Y. Hiwatari, Phys. Rev. A 38, 454 (1988).

[48] J.-N. Roux, J.-L. Barrat, and J.-P. Hansen, J. Phys. Condens. Matter 1, 7171 (1989).

[49] G. Wahnström, Phys. Rev. A 44, 3752 (1991).

[50] N. Bailey, T. Ingebrigtsen, J. S. Hansen, A. Veldhorst, L. Bøhling, C. Lemarchand, A. Olsen, A. Bacher, L. Costigliola, U. Pedersen et al., Sci. Post. Phys. 3, 038 (2017).

[51] See Supplemental Material at http://link.aps.org/ supplemental/10.1103/PhysRevLett.122.175501 for additional fit results and the analysis of interdiffusion and concentration fluctuations, which includes Refs. [52,53].

[52] L. S. Darken, Trans. AIME 175, 184 (1948).

[53] J. R. Manning, Phys. Rev. 124, 470 (1961).

[54] H. E. Stanley, Phase Transitions and Critical Phenomena (Clarendon Press, Oxford, 1971).

[55] F. H. Stillinger, Jr. and R. Lovett, J. Chem. Phys. 48, 3858 (1968).

[56] A. Bhatia and D. Thornton, Phys. Rev. B 2, 3004 (1970).

[57] F. Weysser, A. M. Puertas, M. Fuchs, and T. Voigtmann, Phys. Rev. E 82, 011504 (2010).

[58] J. Horbach, S. K. Das, A. Griesche, M.-P. Macht, G. Frohberg, and A. Meyer, Phys. Rev. B 75, 174304 (2007).

[59] J. Zausch, P. Virnau, K. Binder, J. Horbach, and R. L. Vink, J. Chem. Phys. 130, 064906 (2009).

[60] S. Saito, B. Bagchi, and I. Ohmine, J. Chem. Phys. 149, 124504 (2018). 